\title{
Control of the Radiation Patterns Using Homogeneous and Isotropic Impedance Metasurface
}

\author{
Fan Yang, ${ }^{1}$ Zhong Lei Mei, ${ }^{1}$ and Tie Jun $\mathrm{Cui}^{2}$ \\ ${ }^{1}$ School of Information Science and Engineering, Lanzhou University, Lanzhou 730000, China \\ ${ }^{2}$ State Key Laboratory of Millimetre Waves, School of Information Science and Engineering, Southeast University, \\ Nanjing 210096, China
}

Correspondence should be addressed to Zhong Lei Mei; meizl@lzu.edu.cn and Tie Jun Cui; tjcui@seu.edu.cn

Received 27 September 2014; Revised 10 December 2014; Accepted 10 December 2014

Academic Editor: Sanming Hu

Copyright (C) 2015 Fan Yang et al. This is an open access article distributed under the Creative Commons Attribution License, which permits unrestricted use, distribution, and reproduction in any medium, provided the original work is properly cited.

\begin{abstract}
We propose to control the radiation patterns of a two-dimensional (2D) point source by using impedance metasurfaces. We show that the radiation patterns can be manipulated by altering the surface impedance of the metasurface. Full-wave simulation results are provided to validate the theoretical derivations. The proposed design enjoys novel properties of isotropy, homogeneity, low profile, and high selectivity of frequency, making it potentially applicable in many applications. We also point out that this design can be implemented with active metasurfaces and the surface impedance can be tuned by modulating the value of loaded elements, like resistors, inductors, and capacitors.
\end{abstract}

Recently, the concept of metasurface has been very hot. It is two-dimensional metamaterial rather than the previously proposed bulk metamaterials. Also, it enjoys the properties of low profile and low loss, making it very flexible and easy to implement in the real applications [1-5]. Metasurface can be considered as a patterned thin metallic film, whose electromagnetic behavior may be effectively described by averaged surface impedance [6]. This kind of impedance metasurface has various engineering applications [7]. In 2009, Alù proposed that the impedance surface could be used in the cloaking techniques and gave the name "mantle cloak" [8]. It is ultrathin and can be fabricated easily by printing designed metallic patterns [9]. Later, Zhu et al. successfully realized a metasurface with 360-degree reflection phase turning by controlling the surface impedance [10].

The impedance metasurface also has various applications in the field of antenna. Oliner and Hessel first put forward the comprehensive analysis of propagation of leaky waves on impedance surface [11]. Using this theory, Fong et al. suggested a two-dimensional holographic antenna [12]. This surface can scatter a known input wave and transform it into a desired output by varying the surface impedance. In 2012, the anisotropic impedance metasurface was suggested to control the polarization of the radiated field [13]. More recently, impedance surface was also employed in the threedimensional form [14]. And Hunt et al. extended this surface to microwave imaging by designing far-field radiation mode [15]. These designs of radiation pattern require inhomogeneous or even anisotropic surface impedance, which makes it difficult to implement compared with homogeneous and isotropic one. Here, we exploit the homogeneous and isotropic surface impedance in the control of a radiation pattern. The fact that mantle cloak enjoys many scattering modes motivates us to control the radiation pattern by magnifying a specific mode.

In this work, we propose a very simple model, in which a metasurface radiates under the excitation of a $2 \mathrm{D}$ point source (or a line source). With proper design of surface impedance of the metasurface, a specific pattern can be magnified and becomes dominant in the total field; hence, the model behaves like the radiation of this pattern. Moreover, the radiation pattern can be manipulated by employing different surface impedances. To demonstrate the validity of the theory, full-wave simulation results are given, including those mimicking the radiations of monopole, dipole, and quadripole. In order to demonstrate the flexibility of our design, 
the simulation for two metasurfaces is also made. These simulation results agree very well with the theoretical calculations. Throughout the paper, a time-harmonic factor $e^{\text {jwt }}$ has been used.

Let us first consider a cylindrical metasurface in free space. A $2 \mathrm{D}$ point source (i.e., a line source) is placed near the metasurface, as shown in Figure 1(a). In this situation, the cylindrical metasurface is radiating under the excitation of the line source. The corresponding 2D geometry is illustrated in Figure 1(b), in which $a$ represents the radius of the cylinder, and $I$ represents the current of line source. In analyzing the problem, we set up a cylindrical coordinate system, where the axis of the cylindrical metasurface coincides with the $z$-axis. The coordinate center is set at the center of the metasurface and the coordinates of the line source are $\left(\rho^{\prime}, \varphi^{\prime}\right)$. Then the incident electric field $E^{i}$ can be written as

$$
\begin{aligned}
E^{i}= & E_{z}^{i} \\
= & -\frac{\omega \mu_{0} I}{4} H_{0}^{(2)}\left(k_{0}\left|\vec{r}-\vec{r}^{\prime}\right|\right) \\
= & -\frac{\omega \mu_{0} I_{0}}{4} \\
& \times \begin{cases}\sum_{n=-\infty}^{\infty} A_{s} H_{0}^{(2)}\left(k_{0} \rho^{\prime}\right) J_{n}\left(k_{0} \rho\right) e^{j n\left(\varphi-\varphi^{\prime}\right)} & \rho \leq \rho^{\prime} \\
\sum_{n=-\infty}^{\infty} A_{s} J_{n}\left(k_{0} \rho^{\prime}\right) H_{0}^{(2)}\left(k_{0} \rho\right) e^{j n\left(\varphi-\varphi^{\prime}\right)} & \rho \geq \rho^{\prime},\end{cases}
\end{aligned}
$$

in which $I=A_{s} I_{0}, k_{0}$ is the wave number in free space and $J_{n}(\cdot)$ and $H_{n}^{(2)}(\cdot)$ are cylindrical Bessel function and Hankel function of the second kind, respectively. In our analysis, the excitation is a line source with infinite length, making the electric field parallel to the $z$-axis.

In the presence of the metasurface, the induced scattered field $E_{z}^{s}$ and $H_{\varphi}^{s}$ in the interior and exterior regions of metasurface are expressed as

$$
\begin{gathered}
E_{z}^{s}=-\frac{\omega \mu_{0} I_{0}}{4}\left\{\begin{array}{l}
\sum_{n=-\infty}^{\infty} a_{n} J_{n}\left(k_{0} \rho\right) e^{j n \varphi} \quad \rho \leq a \\
\sum_{n=-\infty}^{\infty} s_{n} H_{0}^{(2)}\left(k_{0} \rho\right) e^{j n \varphi} \quad \rho \geq a,
\end{array}\right. \\
H_{\varphi}^{s}=-\frac{\omega \mu_{0} I_{0}}{4} \cdot \frac{k_{0}}{j \omega \mu_{0}}\left\{\begin{array}{l}
\sum_{n=-\infty}^{\infty} a_{n}\left[J_{n}\left(k_{0} \rho\right)\right]^{\prime} e^{j n \varphi} \quad \rho \leq a \\
\sum_{n=-\infty}^{\infty} s_{n}\left[H_{0}^{(2)}\left(k_{0} \rho\right)\right]^{\prime} e^{j n \varphi} \quad \rho \geq a,
\end{array}\right.
\end{gathered}
$$

in which the common factors $-\omega \mu_{0} I_{0} / 4$ in (1)-(3) are used in order to make the following expressions compact in form. Let us use the boundary condition [8]:

$$
H_{\varphi \mid \rho=a^{+}}-H_{\varphi \mid \rho=a^{-}}=\frac{\widehat{r} \times E_{z \mid \rho=a}}{Z_{s}},
$$

where $Z_{s}$ denotes the surface impedance. It is obvious that $Z_{s}=R_{s}+j X_{s}$, in which $R_{s}$ and $X_{s}$ represent the resistance and reactance of surface impedance, respectively.

Using (1)-(4), we can easily derive the scattering coefficients as

$$
\begin{aligned}
s_{n}= & -\left|\begin{array}{cc}
J_{n}\left(k_{0} a\right) & A_{S} H_{n}^{(2)}\left(k_{0} \rho_{S}\right) J_{n}\left(k_{0} a\right) e^{-j n \varphi_{S}} \\
{\left[J_{n}\left(k_{0} a\right)\right]^{\prime}} & A_{S} H_{n}^{(2)}\left(k_{0} \rho_{S}\right)\left[J_{n}\left(k_{0} a\right)\right]^{\prime} e^{-j n \varphi_{S}}-\frac{j \omega \mu_{0}}{k_{0}} \frac{A_{S} H_{n}^{(2)}\left(k_{0} \rho_{S}\right) J_{n}\left(k_{0} a\right) e^{-j n \varphi_{S}}}{Z_{s}}
\end{array}\right| \\
& \times\left|\begin{array}{cc}
J_{n}\left(k_{0} a\right) & H_{n}^{(2)}\left(k_{0} a\right) \\
{\left[J_{n}\left(k_{0} a\right)\right]^{\prime}} & {\left[H_{n}^{(2)}\left(k_{0} a\right)\right]^{\prime}-\frac{j \omega \mu_{0}}{k_{0}} \frac{H_{n}^{(2)}\left(k_{0} a\right)}{Z_{s}}}
\end{array}\right|
\end{aligned}
$$

In order to magnify a specific pattern for radiations, the corresponding scattering coefficient is set to infinite, which can be easily achieved by setting the denominator of (5) to zero. This leads to the expression of the surface impedance as

$$
Z_{s}=-\frac{\pi \omega \mu_{0} a}{2} J_{n}\left(k_{0} a\right) H_{n}^{(2)}\left(k_{0} a\right),
$$

which is used in the calculation of surface impedance for the corresponding radiation of a monopole, a dipole, a quadripole, and so forth. This is easily obtained by setting the order $n$ in the above equation to $0,1,2, \ldots$, respectively.

Using Huygens' principle, the electric field in the far-field region is calculated to determine the scattering width $\sigma$. Since the problem in this work is $2 \mathrm{D}$, we only need to figure out $\sigma_{2 \mathrm{D}}$ [16], which is given as follows:

$$
\begin{aligned}
\sigma_{2 \mathrm{D}}=\left(k_{0} \mid \widehat{r}_{0} \times \oint_{C}\right. & {\left[\left(\widehat{n} \times \vec{E}_{c}\right)-\eta_{0} \widehat{r}_{0} \times\left(\widehat{n} \times \vec{H}_{c}\right)\right] } \\
& \left.\times\left.\exp \left(i k \vec{r}^{\prime} \cdot \widehat{r}_{0}\right) d l\right|^{2}\right) \times\left(4\left|\vec{E}^{i}\right|^{2}\right)^{-1},
\end{aligned}
$$

where $\vec{E}_{c}$ and $\vec{H}_{c}$ are the EM field on the integration contour $C, \widehat{r}_{0}$ is the unit vector of the scattering direction, $\vec{r}^{\prime}$ is the 


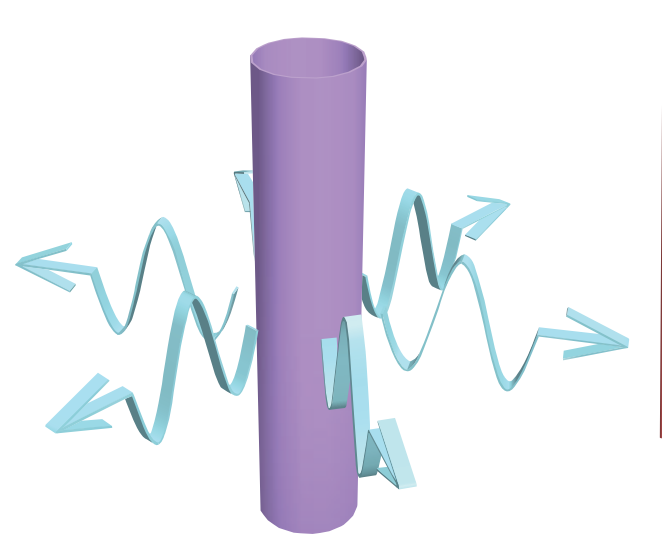

(a)

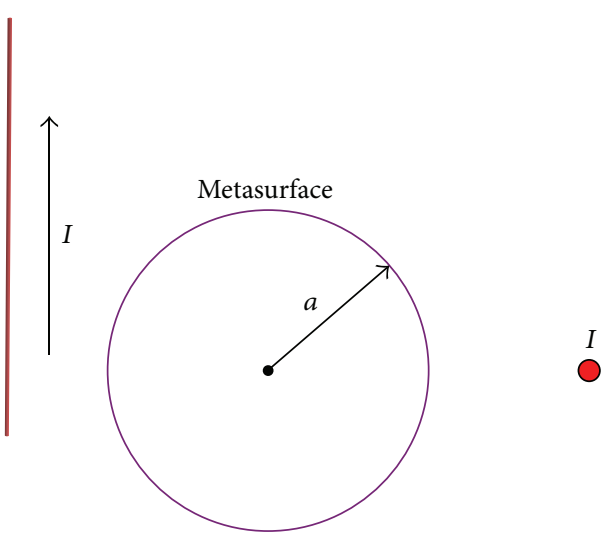

(b)

FIGURE 1: Principle of the proposed model with a cylindrical metasurface and a line source (color online). (a) The three-dimensional depiction. (b) The two-dimensional geometry of the cross section.

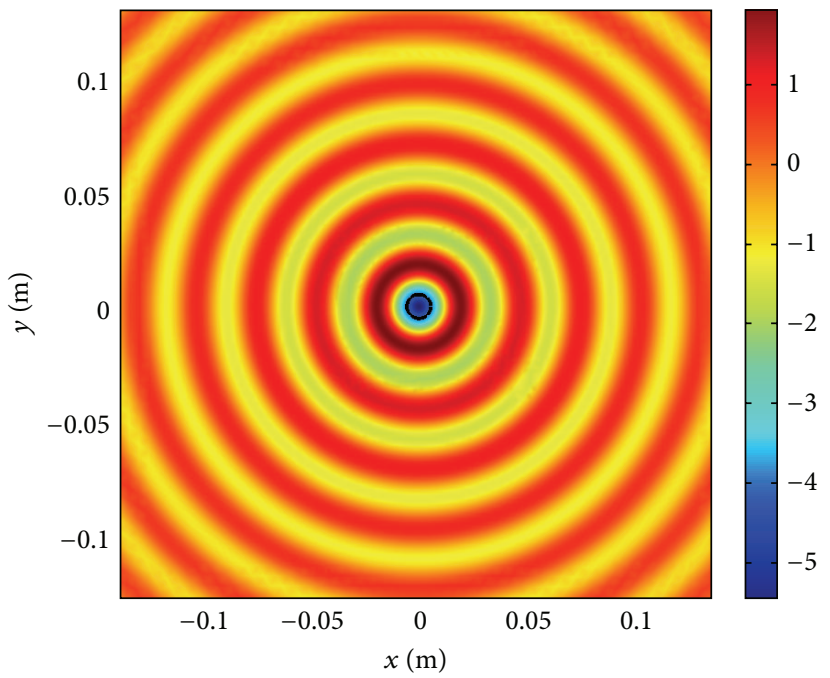

(a)

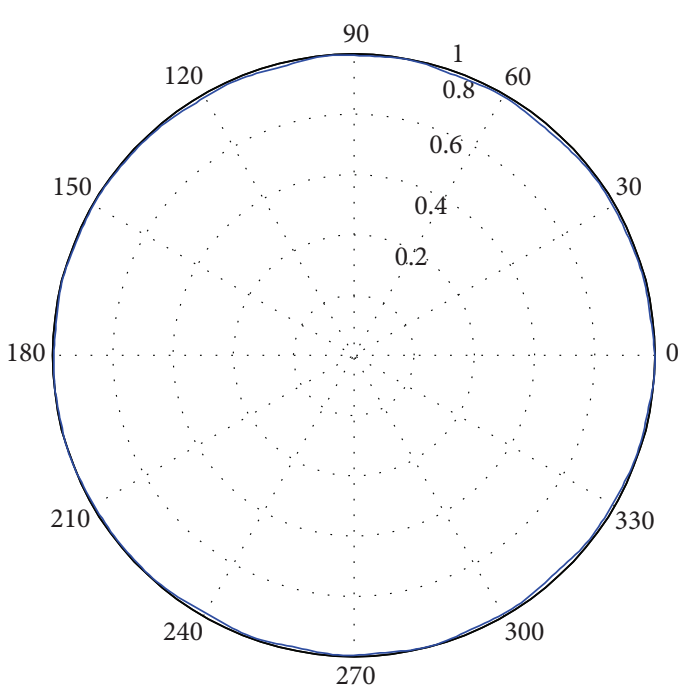

(b)

Figure 2: The simulation result of a monopole radiation (color online). (a) The electric field distribution in the $x-y$ plane. (b) The far-field radiation pattern with a circular shape.

position vector on the contour $C$, and $\eta_{0}$ is the free space wave impedance. So we can calculate the radar cross section based on this equation. Note that the integration contour $C$ can be chosen in near-field when there is no source in exterior region of this contour. To generally measure the overall scattering width versus frequency, the total scattering width is used [9, 17]:

$$
\sigma_{\text {total }}=\frac{4}{k_{0}} \sum_{n=-\infty}^{n=\infty}\left|s_{n}\right|^{2}
$$

It is defined as the radio of the energy across a closed surface that encloses the scatterer to the incident irradiance.

Next we perform full-wave simulations to verify the proposed theory, in which the working frequency is set as $10 \mathrm{GHz}$. Using (6), we first consider the case of a monopole radiation. In our simulation, the radius of the cylindrical metasurface is set to $\lambda_{0} / 5$ ( $\lambda_{0}$ represents the wavelength in free space) and the polar coordinates of the excitation source $\left(\rho_{s}, \varphi_{s}\right)$ are $\left(\lambda_{0}, 0\right)$. The values of $I_{0}$ and $A_{s}$ are both set as 1 . The calculation result shows that the surface impedance for the monopole radiation is simply $Z_{s}=(-307.2+125.3 j) \Omega$. Figure 2 demonstrates the simulation result of the monopole radiation, in which Figure 2(a) illustrates the electric field distribution in the $x-y$ plane. It is obvious that the scattered field by the metasurface looks like a monopole radiation. Moreover, it is clear that the incident field of line excitation becomes negligible when the monopole pattern is magnified.

In order to quantify our simulation result, the electric field on a centered circle with radius $0.1 \mathrm{~m}$ is extracted from the simulation result. Then the scattering width $\sigma_{2 \mathrm{D}}$ is calculated using the proposed method, and the far-field radiation pattern is given in the polar diagram in Figure 2(b). Obviously, the radiation pattern is nearly a circle, which 


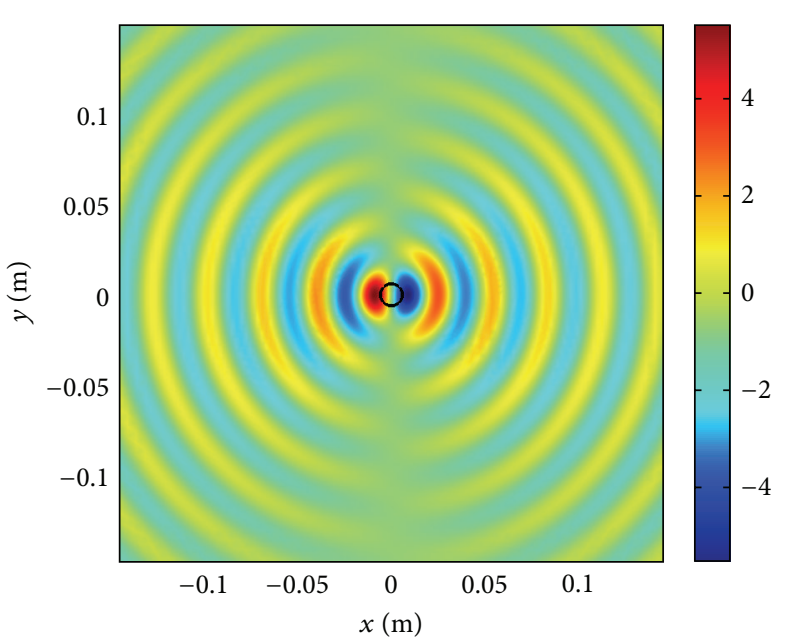

(a)

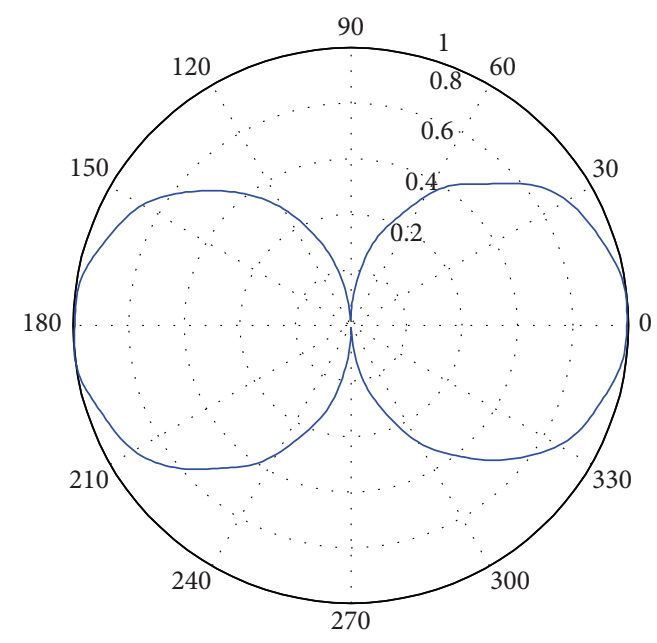

(b)

FIgURE 3: The simulation result for a dipole radiation (color online). (a) The electric field distribution in the $x$ - $y$ plane. (b) The far-field radiation pattern with a " $\infty$ " shape.

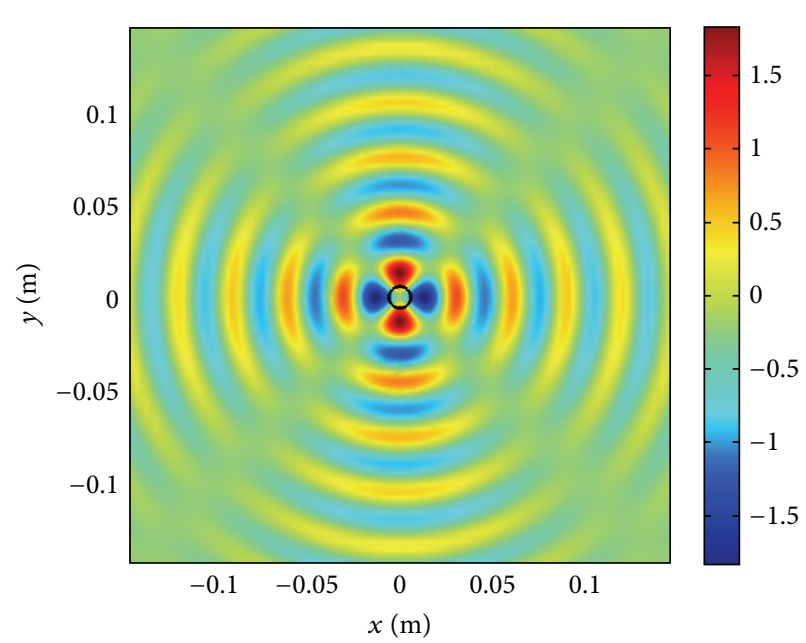

(a)

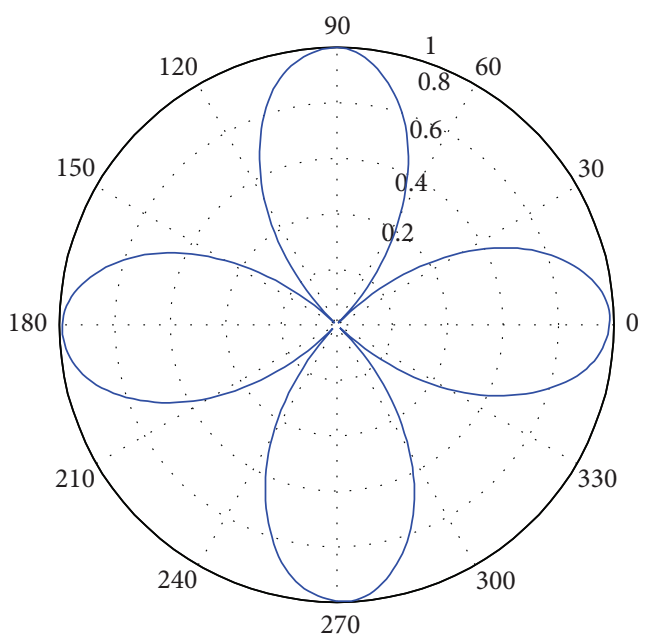

(b)

FIGURE 4: The simulation result of a quadripole radiation (color online). (a) The electric field distribution in the $x-y$ plane. (b) The far-field radiation pattern with a petal shape.

quantitatively verifies the correctness of the theoretical analysis. Note that a little discrepancy exists in the far-field radiation pattern, which is due to some disturbance from the remaining patterns. However, these disturbances are ignorable when compared to the designed radiation pattern, making the metasurface behave like a monopole.

Then we choose $n=1$, and the surface impedance of metasurface is calculated as $Z_{s}=(-195.2-220.9 j) \Omega$, which corresponds to the case when the dipolar pattern is magnified. The performance for this metasurface is presented in Figure 3. Similarly, both the near electric field distribution and far-field radiation pattern are studied. The simulation result illustrated in Figure 3(a) shows that the radiation of this metasurface looks like a dipole, in which the excitation source also becomes ignorable. The quantitative depiction of far-field radiation pattern is given in Figure 3(b). Note that the curve of radiation pattern is not a rigid " $\infty$ " shape because some other patterns have also been magnified, affecting the total radiation field. But these patterns are overwhelmed by the dominant dipole field, making the total radiation pattern behave like a dipole.

We continue to consider the case of quadripole, and the corresponding value of surface impedance is obtained by setting $n=2$, which yields $Z_{s}=(-22.2-152.2 j) \Omega$. Figure 4 shows the simulation results of the quadripole case, in which Figure 4(a) is the near-field distribution and Figure 4(b) gives the corresponding far-field radiation pattern. Through the change of surface impedance, the metasurface can truly 


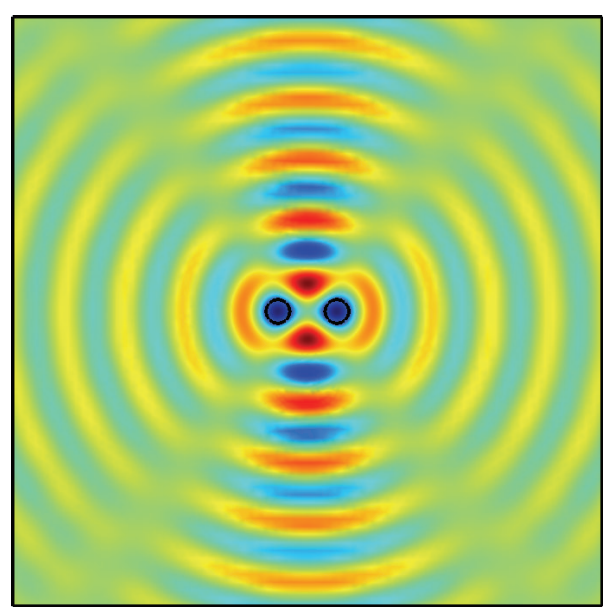

(a)

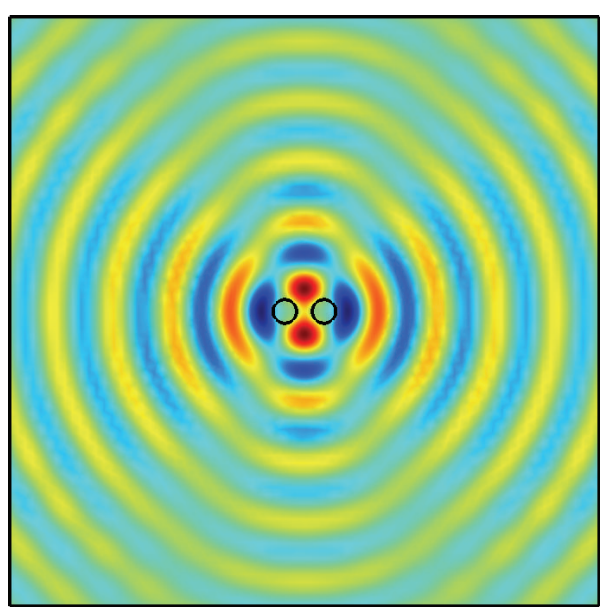

(c)

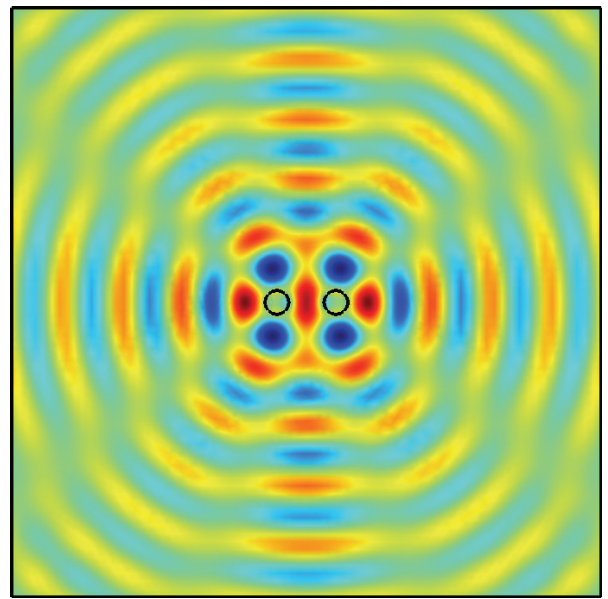

(e)

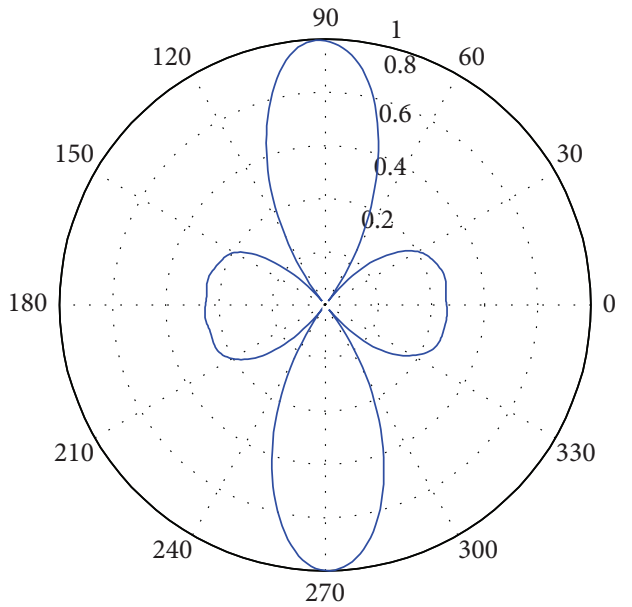

(b)

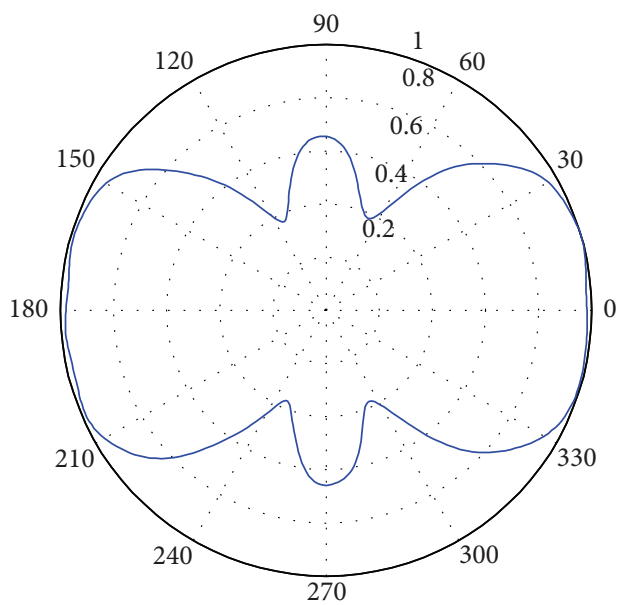

(d)

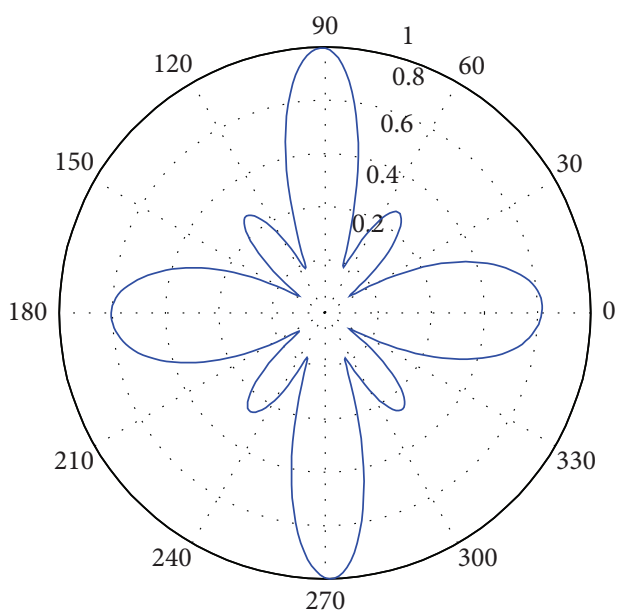

(f)

Figure 5: ((a), (c), and (e)) The electric field distribution in the $x$ - $y$ plane, where mode $n$ equals 0,1 , and 2, respectively (color online). ((b), (d), and (f)) The corresponding far-field radiation pattern of (a), (c), and (e). 


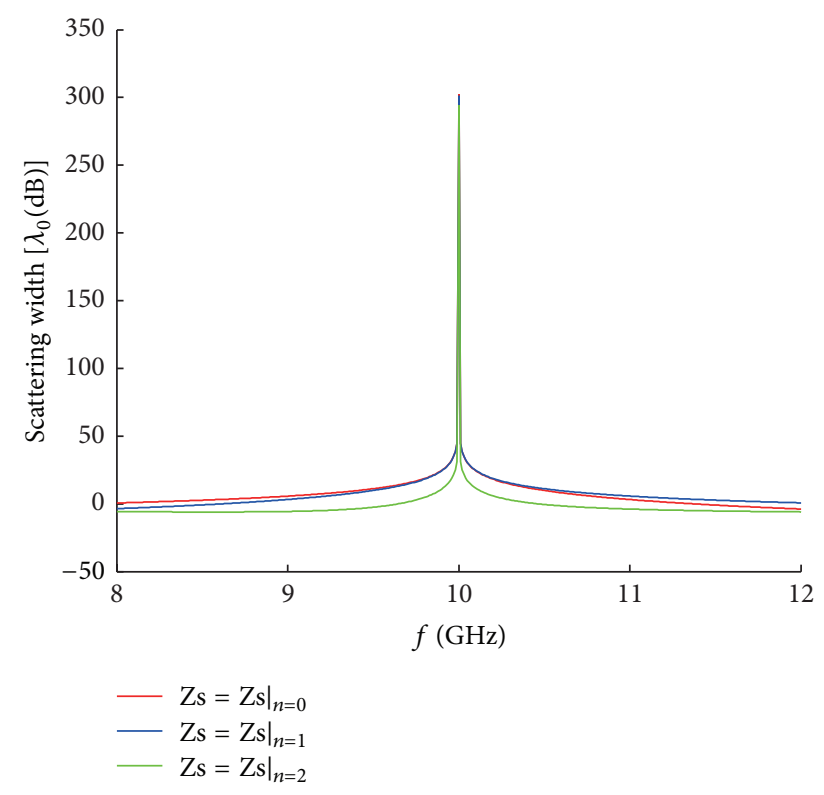

FIGURE 6: The total scattering widths versus frequency, in which different surface impedance is considered, including the corresponding monopole, the dipole, and the quadripole cases (color online).

radiate like a quadripole, whose far-field pattern has a typical petal shape.

Likewise, other radiation patterns, like sextupole and octupole radiations, can also be achieved by setting the order $n$ to 3 and 4, respectively. And from the calculation results, we can easily reach the conclusion that the proposed metasurface is active due to the fact that surface resistance has a negative value.

However, the proposed metasurface can be regarded as a basic unit. And we can put several units to create many other kinds of radiation patterns. In the following part, the simulations of radiation pattern when there are two cylindrical metasurfaces with same parameters are given, in which three cases mean mode $n$ equals 0,1 , and 2 , respectively. The simulation results are shown in Figure 5. Additionally, more attainable radiation patterns can be realized by different combination of these units.

Using (8), the variations of total scattering widths versus frequency are calculated, as shown in Figure 6, including the cases of monopole, dipole, and quadrupole. This figure demonstrates that the scattering width is very sensitive to the deviation of frequency from $10 \mathrm{GHz}$. In other words, this metasurface is selective in scattering frequency, which can avoid the disturbance of other frequencies in the real applications.

Recently, active devices have been suggested to realize some negative electromagnetic parameters, which may not be attainable in nature. In our work, a negative resistor model was put forward to meet the negative conductivity requirement of an exterior cloak [18]. This model consists of a source and two resistors in order to make the input current have a negative linear relation with voltage. Also, loading of negative impedance converter (NIC) elements has been proposed as a way to realize an active metasurface [19]. These loading elements can provide an effective negative capacitance. Based on these ideas, it can be expected that a metasurface with negative resistance can also be realized in a similar manner.

With regard to control of surface impedance, we point out that its value can be changed by modulating the parameter of some loaded elements, like resistors, inductors, or capacitors. For example, in Liu's work, the surface impedance is tuned by voltage-modulation of a varactor diode [20].

In summary, we have shown that a designed radiation pattern can be achieved by using a line source to excite a metasurface. By selecting proper surface impedance, the radiation pattern of total field can be a magnified monopole, dipole, quadripole, and so forth. This makes it possible to build up a multipolar antenna system with the simple configuration. The most important property of the model is that its radiation pattern is controlled by simply changing the surface impedance rather than modifying the geometrical shape. And this surface impedance is homogeneous and isotropic in contrast with previous holographic impedance surface. Moreover, this model is ultrathin and selective in the radiation frequency.

\section{Conflict of Interests}

The authors declare that there is no conflict of interests regarding the publication of this paper.

\section{Acknowledgments}

This work was supported in part by National High Tech (863) Projects (Grants no. 2012AA030402 and 2011AA010202), in part by the 111 Project (111-2-05), and in part by the National Science Foundation of China (Grants no. 60990320 and 60990324). Zhong Lei Mei acknowledges the Open Research Funds of State Key Laboratory of Millimeter Waves (Grant no. K201409) and Fundamental Research Funds for the Central Universities (Grant nos. LZUJBKY-2013-k06, LZUJBKY2014-43, and LZUJBKY-2014-237).

\section{References}

[1] N. Yu, P. Genevet, M. A. Kats et al., "Light propagation with phase discontinuities: Generalized laws of reflection and refraction," Science, vol. 334, no. 6054, pp. 333-337, 2011.

[2] N. Yu, P. Genevet, F. Aieta et al., "Flat optics: controlling wavefronts with optical antenna metasurfaces," IEEE Journal of Selected Topics in Quantum Electronics, vol. 19, no. 3, Article ID 4700423, 2013.

[3] C. L. Holloway, E. F. Kuester, J. A. Gordon, J. O'Hara, J. Booth, and D. R. Smith, "An overview of the theory and applications of metasurfaces: The two-dimensional equivalents of metamaterials," IEEE Antennas and Propagation Magazine, vol. 54, no. 2, pp. 10-35, 2012.

[4] A. V. Kildishev, A. Boltasseva, and V. M. Shalaev, "Planar photonics with metasurfaces," Science, vol. 339, no. 6125, p. 1289, 2013.

[5] N. Yu and F. Capasso, "Flat optics with designer metasurfaces," Nature Materials, vol. 13, no. 2, pp. 139-150, 2014. 
[6] S. Tretyakov, Analytical Modeling in Applied Electromagnetics, Artech House, Boston, Mass, USA, 2003.

[7] B. A. Munk, Frequency Selective Surface: Theory and Design, John Wiley \& Sons, New York, NY, USA, 2000.

[8] A. Alù, "Mantle cloak: invisibility induced by a surface," Physical Review B, vol. 80, Article ID 245115, 2009.

[9] P. Y. Chen and A. Alu, "Mantle cloaking using thin patterned metasurfaces," Physical Review B, vol. 84, Article ID 205110, 2011.

[10] B. O. Zhu, J. M. Zhao, and Y. J. Feng, "Active impedance metasurface with full 360。 reflection phase tuning," Nature Scientific Reports, vol. 3, article 3059, 2013.

[11] A. Oliner and A. Hessel, "Guided waves on sinusoidally-modulated reactance surfaces," IRE Transactions on Antennas and Propagation, vol. 7, no. 5, pp. 201-208, 1959.

[12] B. H. Fong, J. S. Colburn, J. J. Ottusch, J. L. Visher, and D. F. Sievenpiper, "Scalar and tensor holographic artificial impedance surfaces," IEEE Transactions on Antennas and Propagation, vol. 58, no. 10, pp. 3212-3221, 2010.

[13] R. Quarfoth and D. Sievenpiper, "Broadband unit-cell design for highly anisotropic impedance surfaces," IEEE Transactions on Antennas and Propagation, vol. 62, no. 8, pp. 4143-4152, 2014.

[14] D. J. Gregoire, “3-D conformal metasurfaces," IEEE Antennas and Wireless Propagation Letters, vol. 12, pp. 233-236, 2013.

[15] J. Hunt, T. Driscoll, A. Mrozack et al., "Metamaterial apertures for computational imaging," Science, vol. 339, no. 6117, pp. 310313, 2013.

[16] C. Li and F. Li, "Two-dimensional electromagnetic cloaks with arbitrary geometries," Optics Express, vol. 16, no. 17, pp. 1341413420, 2008.

[17] C. F. Bohren and D. R. Huffman, Absorbtion and Scattering of Light by Small Particles, Wiley, New York, NY, USA, 1983.

[18] F. Yang, Z. L. Mei, X. Y. Yang, T. Y. Jin, and T. J. Cui, "A negative conductivity material makes a dc invisibility cloak hide an object at a distance," Advanced Functional Materials, vol. 23, no. 35, pp. 4306-4310, 2013.

[19] P. Y. Chen, C. Argyropoulos, and A. Alu, "Broadening the cloaking bandwidth with non-foster metasurfaces," Physical Review Letters, vol. 111, Article ID 233001, 2013.

[20] S. Liu, H.-X. Xu, H. C. Zhang, and T. J. Cui, “Tunable ultrathin mantle cloak via varactor-diode-loaded metasurface," Optics Express, vol. 22, no. 11, Article ID 13403, 2014. 

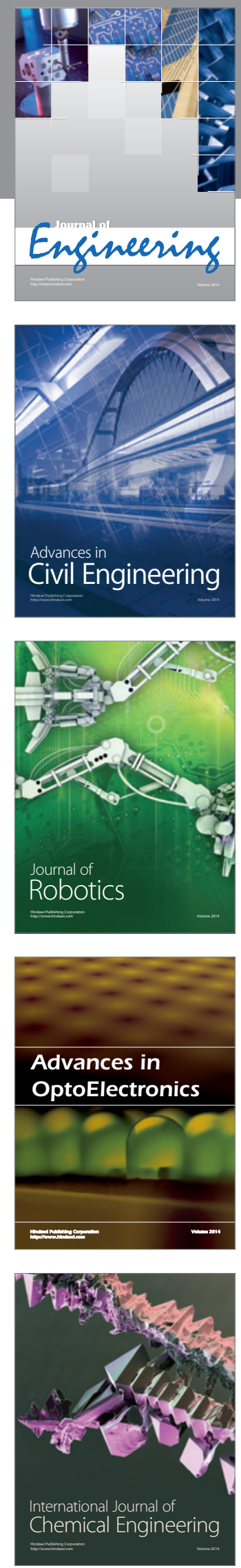

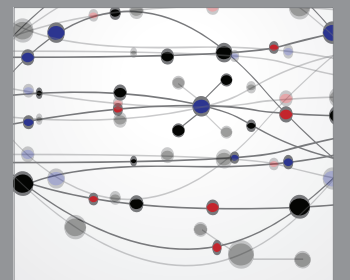

The Scientific World Journal
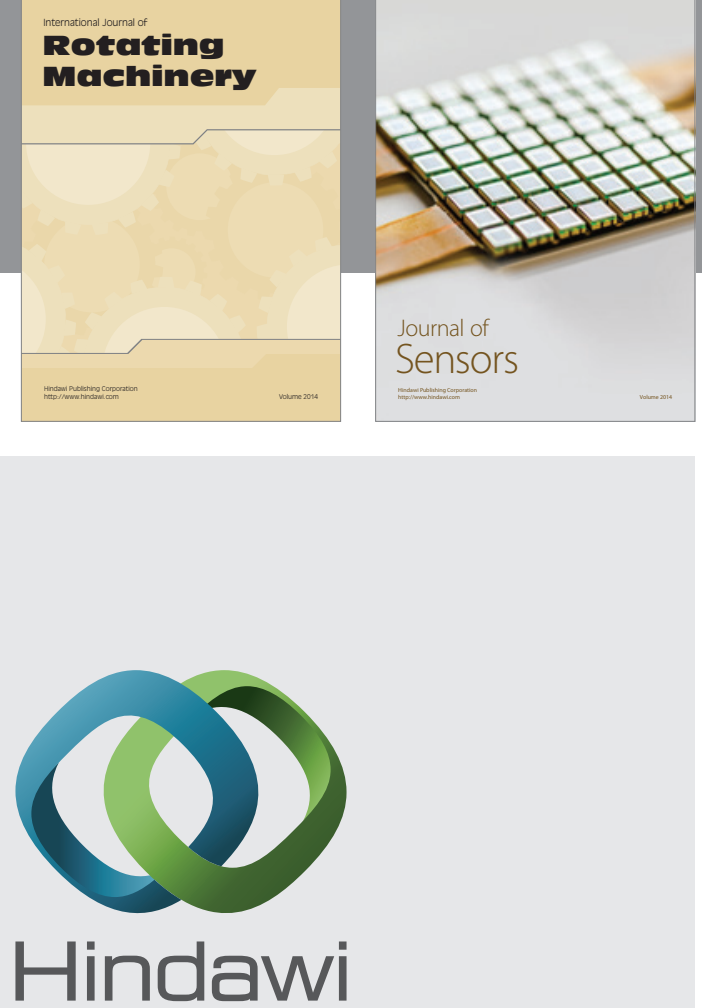

Submit your manuscripts at http://www.hindawi.com
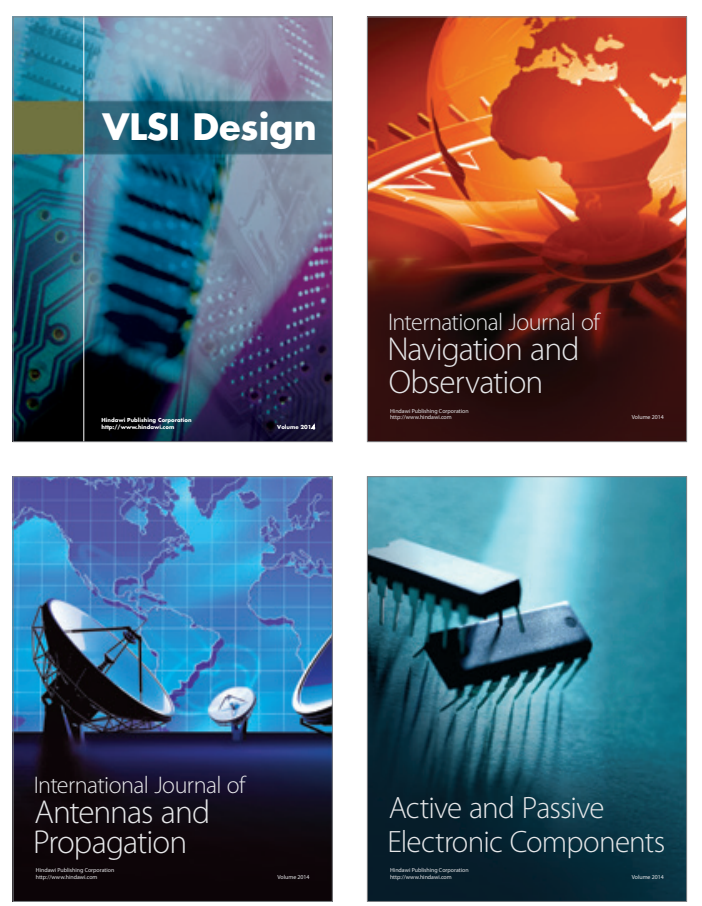
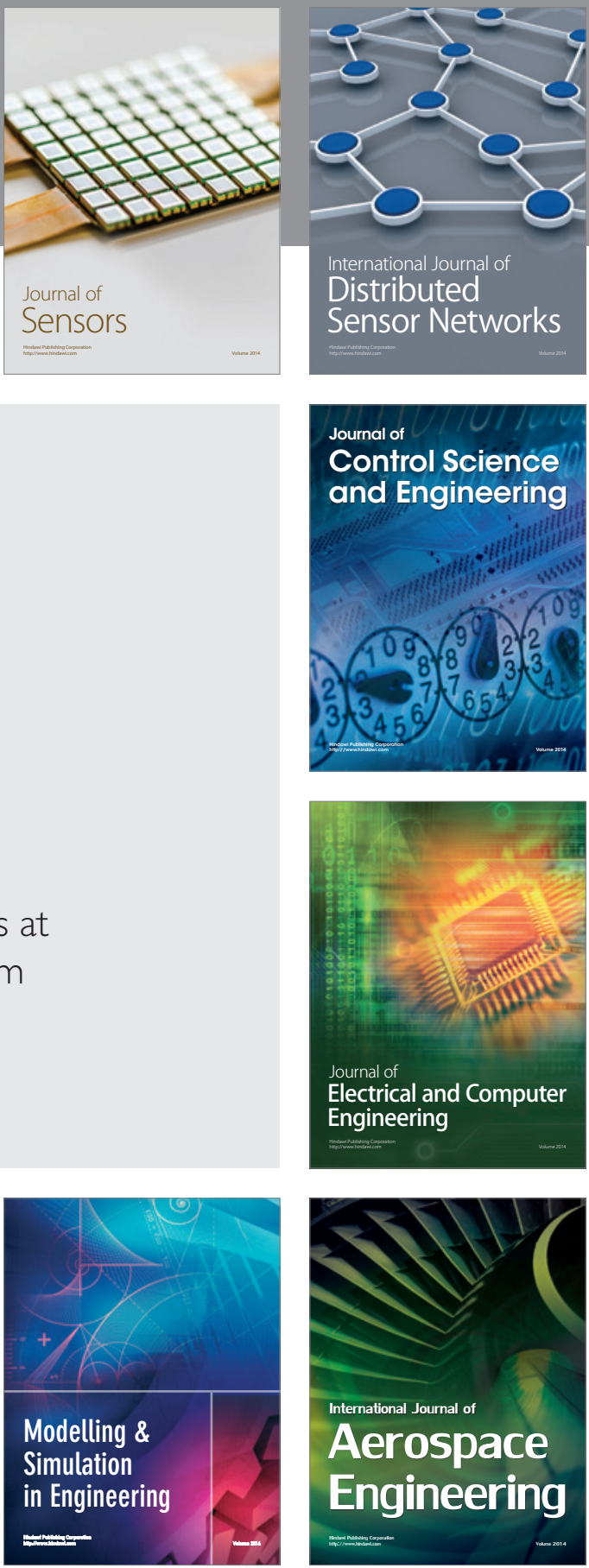

Journal of

Control Science

and Engineering
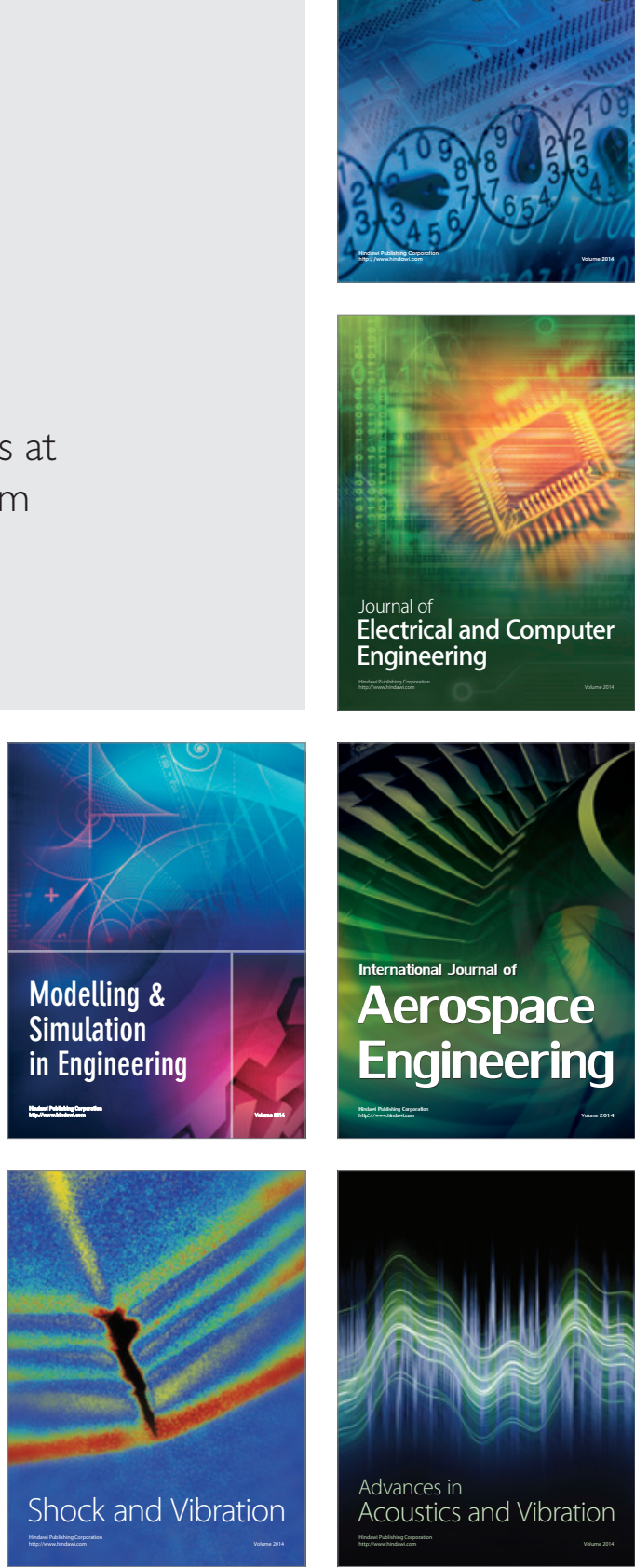